\title{
Use of ethinylestradiol/drospirenone combination in patients with the polycystic ovary syndrome
}

\author{
Ruchi Mathur' \\ Olga Levin' \\ Ricardo Azziz' \\ 'Cedars-Sinai Medical Center, Los \\ Angeles, CA, US
}

\begin{abstract}
Polycystic ovary syndrome (PCOS) is one of the most common endocrine/metabolic disorders found in women, affecting approximately 105 million women worldwide. It is characterized by ovulatory dysfunction, often presenting as oligomenorrhea or amenorrhea and either clinical or biochemical hyperandrogenism. Combined oral contraceptive (COC) therapy has long been a cornerstone of care for women with PCOS. COC therapy often provides clinical improvement in the areas of excessive hair growth, unpredictable menses, acne, and weight gain. One of the main issues in COC therapy is choosing the most appropriate progestin component to provide the greatest anti androgenic effects. Drospirenone, a relatively new progestin, has shown benefit in the PCOS population when used in conjunction with ethinyl estradiol. We now review the role of COCs in PCOS, focusing specifically on drospirenone. Controversy over metabolic effects of COCs in PCOS is also discussed.
\end{abstract}

Keywords: polycystic ovary syndrome, PCOS, oral contraceptives, drospirenone, treatment

\section{Introduction}

Polycystic ovary syndrome (PCOS) is one of the most common endocrine/metabolic disorders found in women. While the definition remains a point of controversy for some, PCOS is characterized by ovulatory dysfunction, usually presenting as oligomenorrhea or amenorrhea, and either clinical or biochemical hyperandrogenism (Azziz et al 2006). The hyperandrogenism can result in hirsutism, oligo-amenorrhea, acne, and alopecia.

The prevalence of PCOS (based on the NIH 1990 criteria) in women of reproductive age is approximately $6.5 \%-8.0 \%$ (Michelmore et al 1999). This encompasses approximately 5 million women in the United States and 105 million women worldwide.

Clinically, PCOS is a heterogeneous disorder of functional androgen excess and the features of PCOS can run through a spectrum of severity. The clinical features may differ according to ethnicity, environmental factors, and medical co-morbidities (Table 1). Most features can be elicited by performing a precise history and physical examination.

The primary aspects of PCOS that require treatment are oligo-amenorrhea, hyperandrogenism, and metabolic concerns such as insulin resistance (Lobo 2006). While the majority of these patients show functional hyperandrogenism clinically, studies have found conflicting results (Pugeat et al 1993; Knochenhauer et al 1998; Legro et al 1998; Laven et al 2002; Azziz et al 2006) regarding absolute androgen levels. The ovaries are the primary site of implication in excessive production of androgens in PCOS. Theca cells (under the influence of lutenizing hormone [LH]) are overactive in steroidogenesis, subsequently providing excess androgens to function as a substrate for estradiol production through the process of aromatization (Azziz et al 2006). LH hypersecretion by the pituitary gland may be present or concentrations of LH may be elevated due to increased amplitude and frequency of LH pulse. 
Table I Clinical manifestations of polycystic ovary syndrome

Menstrual abnormalities (including oligo-amenorrhea, polymenorrhea, and dysfunctional uterine bleeding)

Excess facial and body terminal hair growth, and hirsutism

Seborrhea and acne

Alopecia

Obesity and central (visceral) fat distribution

Acanthosis nigricans and acrochordons

Polycystic ovaries

Modified with permission from Azziz R, Nestler JE, et al eds. 2006. Androgen excess disorders in women, polycystic ovary syndrome and other disorders. 2nd ed.Totowa, New Jersey: Humana Press.

This is most often accompanied by a decrease in circulating follicle stimulating hormone (FSH). Both the $\mathrm{LH}$ and the FSH profiles are thought secondary to a fundamental increase in GnRH from the hypothalamus which ultimately favors the gene expression of LHb over FSHb (Azziz et al 2006). Insulin resistance with compensatory increases in circulating insulin levels may be present as well. The biochemical abnormalities noted above often result in anovulation. PCOS is the most common cause of anovulatory infertility (Laven et al 2002).

Hirsutism, or excessive hair growth in a male-like pattern, can be observed in approximately $75 \%$ of women with PCOS of white or black race (Azziz et al 2004). Areas of excessive hair growth include the face, anterior chest, midline abdomen area, and pubic region. There can be significant variation in presentation of hirsutism depending on genetics and race. Clinical assessment is based on a visual scoring system (Ferriman-Gallwey 1961). Therapies for treating hair growth range from cosmetic procedures, such as laser therapy, to medications. Antiandrogens, including spironolactone, cyproterone acetate (CPA), flutamide, and finasteride, are commonly prescribed for this condition, often in conjunction with other therapies, such as oral contraceptives (Venturoli et al 1999; Moghetti et al 2000).

Oligo-amenorrhea and the resultant fertility problems can be treated with agents such as, clomiphene, letrazole, metformin, and gonadotropin therapy, which induce ovulation. This mode of treatment takes into account the concept that androgenic problems typical in women with PCOS are an effect of concomitant metabolic problems such as insulin insensitivity. Thus, drugs that treat the insulin insensitivity, such as metformin, should also be beneficial in the treatment of hyperandrogenism and restore ovulation in women suffering from PCOS. Another common mode of treatment of hyperandrogenism related to PCOS is oral contraceptives (used to suppress ovarian activity) often in conjunction with an anti-androgen agent (Lobo 2006).

\section{Oral contraceptives in PCOS}

Combined oral contraceptives (COC) are a common treatment for PCOS and have a long history of use in this group of patients. Adolescents and women with PCOS have a number of different clinical symptoms that play a role in their daily quality of life. Excessive hair growth, irregular and unpredictable menses, acne and weight gain are all part of the clinical constellation seen in this population. COC has long been considered a first line of therapy for women experiencing these symptoms and who do not wish to get pregnant. Publications from the 1960s (Mauvais-Jarvis et al 1965) reported the inhibition of androgens with the use of "ovulation inhibitors". Since then multiple reports have confirmed this finding particularly with the use of antiandrogenic progestin components.

The key mechanism of COC action is folliculogenesis inhibition through either suppression of pituitary gonadtorophin secretion or direct influence on ovarian function when the COC agent has weaker anti-gonadotrapin activity. COC can also lead to a decrease in circulating albumin levels, leading to a decrease in dehydroepiandrosterone sulphate (DHEAS), which is strongly bound to albumin (Carlstrom et al 2002) and androgen production (Vrbikova and Cibula 2005) and a decrease in peripheral androgen production through an inhibition of $5 \alpha$ reductase in the skin and a subsequent decrease in dihydrotestosterone levels (Cassidenti et al 1991). The combined effect is an overall decrease in gonadotropins and resulting androgens, which is a major goal of PCOS treatment. In addition, the estrogen component of COC increases sex hormone binding globulin (SHBG) production by the liver (Wiegratz et al 2003), which in turn reduces the circulating levels of free testosterone and its bioavailability.

One of the main issues in choosing COC for the treatment of PCOS is finding the most appropriate progestin component to provide the greatest anti androgenic effects. The criteria for oral contraceptive selection has not been thoroughly evaluated. It should be based on currently available clinical data, as well as on patient tolerance and response. Selection of a COC is based on 3 main variables: the type of estrogenic compound used, the type of progestin compound used, and the dosage of each when combined. In general, while the use of a monophasic compound may be preferred by the patient for its ease of use, the choice of a monophasic, biphasic or triphasic compound does not have any significant effect on androgen production (Vrbikova and Cibula 2005). Today, the vast majority of COCs contain ethinyl estradiol (EE) as their estrogen, and thus only the type of progestin and the dosages of the steroids contained remain to be assessed. 
The dose of EE varies between 15 and $50 \mu \mathrm{g}$, and the effects on the pituitary-ovarian axis occur in a dose dependent fashion. A daily dose of $30-35 \mu \mathrm{g}$ of EE is appropriate for suppression of ovarian folliculogenesis in most women. EE is responsible for increasing circulating levels of SHBG Wiegratz (Wiegratz et al 2003). As a result, its overall androgenic potential is in part dependent on the progesterone it is combined with, and the progesterone effect on SHBG. Wiegratz and colleagues and van der Vange et al have shown that combining the same dose EE with different progesterone compounds resulted in significantly different levels of SHBG (van der Vange et al 1990; Wiegratz et al 2003).

A number of different progestins are available in combination oral contraceptives. Table 2 lists the most common progestins available and their androgenic profiles. Cyproterone acetate (CPA) is a commonly used progestin for the treatment of hirsutism in adolescence and beyond (Creatsas et al 2000), as it exhibits both progestogenic and significant antiandrogenic properties. As all progestins, it inhibits LH, and subsequently the production of androgens by the ovarian theca cells, and increases the hepatic clearance of testosterone. It also competes at the receptor sites with androgens and thus exerts an antiandrogenic influence. We should note that the amount of CPA present in COC $(2 \mathrm{mg})$ exerts a modest effect on hirsutism, and often the addition of higher doses of the steroid (12.5-80 mg/day) are required for maximum suppression of hair growth (Hammerstein et al 1975; Barth et al 1991). CPA also demonstrates mild anti-glucorticoid effects which may become more apparent at higher doses.

A newer form of progestin, drospirenone, has been recently introduced, and is marketed as part of a COC (Yasmin ${ }^{\circledR}$ and Yaz $^{\circledR}$ in the United States), in combination with EE. The next section of this article will focus on the progestin drospirenone, and its use in patients with PCOS.

Table 2 Currently available progestins and their androgenic effects

\begin{tabular}{ll}
\hline Progestin & Androgenic effect \\
\hline Levonorgestrel & High \\
Norgestrel & High \\
Norethindrone & Medium \\
Norethindrone acetate & Medium \\
Ethynodiol diacetate & Low \\
Norgestimate & None \\
Desogestrel & None \\
Drosperinone & Antiandrogenic \\
\hline
\end{tabular}

\section{Drospirenone - a progestin with differences}

Drospirenone, in conjunction with EE, acts to suppress gonadotropins. This is achieved through inhibition of ovulation as described above. Drospirenone has a bioavailability of $76 \%$ with approximately $20 \%$ excreted through the feces, and $45 \%$ through the renal system. The half-life of drospirenone is 30 hours, which is slightly longer than the half-life of EE (24 hours).

Drospirenone differs from other progestins currently available in other COC compounds, as it exhibits both mineralocorticoid effects and antiandrogenic effects. Part of the novel nature of drospirenone stems from it being structurally and functionally analogous to spironolactone (Figure 1). Spironolactone is an aldosterone antagonist, and a potassium-sparing diuretic. Because of this structural and functional similarity with spironolactone, drospirenone also exhibits anti-mineralocorticoid activity. This property counteracts the estrogen stimulated activity of the reninangiotensin-aldosterone system, which can influence the regulation of water and electrolyte balance. Because of the possibility that potassium levels may increase, drospirenone should not be used in patients with kidney, liver or adrenal

(a)

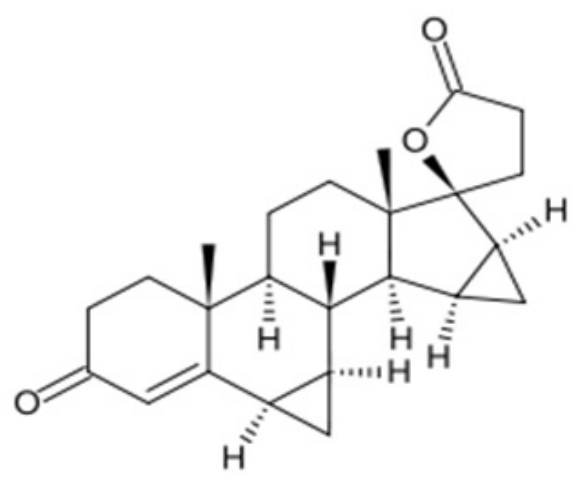

(b)

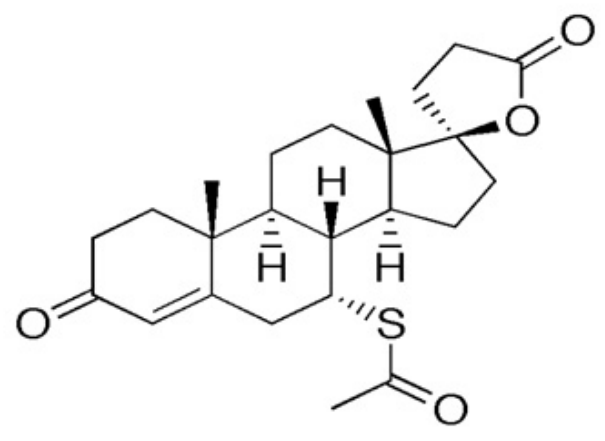

Figure I Structure of drospirenone (I a) and spironolactone (I b). 
disease. In addition, any patient on other medications that can raise potassium levels (such as ACE inhibitors, angiotensin II receptor antagonists, NSAIDS, potassium-sparing diuretics, and heparin) should be cautioned. This anti-mineralocorticoid activity may also contribute to less water retention as well as less breast swelling and tenderness in women using this form or progestin compared to others. Preclinical studies in animals and in vivo have shown that drospirenone has no androgenic, estrogenic, glucocorticoid or anti-glucocorticoid activity (product monograph). Preclinical studies have shown anti-androgenic activity. Due to its anti-androgen effect, drospirenone can also be used as adjunct treatment for hirsutism, and may be a progestin of choice in women who complain of excessive hair growth.

\section{Drospirenone in combination with EE}

Drospirenone is taken orally in preparations with EE. At present, 2 combinations are available for use in the United States: 1) Yasmin ${ }^{\circledR}$ containing $3 \mu \mathrm{g}$ of drospirenone in combination with $30 \mu \mathrm{g}$ for EE per tablet; and 2) Yaz $^{\circledR}$ which contains $3 \mu \mathrm{g}$ of drospirenone and $20 \mathrm{mcg}$ of EE per tablet. As a note, it is also available in a preparation to manage post-menopausal symptoms. Angeliq ${ }^{\circledR}$ was approved by the US FDA for oral use at a dose of $0.5 \mu \mathrm{g}$ drospirenone per day combined with $1 \mu \mathrm{g}$ estradiol for treatment of menopausal symptoms.

Adverse effects may include a change of weight, increase in migraine activity, depression, and nausea. As with most COC there may also be breast swelling and tenderness (though usually to a lesser extent as discussed previously) as well as break-through-bleeding. In addition to the specific drug interactions mentioned above related to the antimineralocortcoid effect, the same interactions should be considered as with the use of any COC. These include but are not limited to interactions with antipsychotics, cyclosporins, dilantin, steroids, and rifampin.

As with all COC this medication is contraindicated in patients with hepatic dysfunction, and in those who smoke and those who have a history of coagulopathy, DVT or stroke. Other contraindications for the use of COC also apply to the products containing drospirenone such as carcinoma of the breast or endometrium, and undiagnosed genital bleeding. Patients over the age of 35 may also be at higher risk or complications such as thrombophlebitis and should be cautioned as such. The use of drospirenone in premenstrual women is not approved.

When drospirenone in combination with $30 \mu \mathrm{g}$ of EE per tablet is compared to $3 \mu \mathrm{g}$ of drospirenone in combination with of EE per tablet, the latter may have less of a hemostatic impact (Kluft et al 2006).

\section{Drospirenone and PCOS}

COCs containing drospirenone are a viable and potentially preferred option for use in the treatment of PCOS and its related symptoms. The efficacy of COCs containing drospirenone as the progestin agent has recently become the topic of much study. Drospirenone has proven to be effective in decreasing hirsutism and testosterone levels and, more significantly, increasing sex hormone binding globulin (SHBG) levels in women who have PCOS.

Pehlivanov and Mitkov performed an open study in which 20 women with PCOS (Rotterdam criteria 2003) were enrolled (Pehlivanov and Mitkov 2007). All women received $30 \mu \mathrm{g}$ EE and $3 \mathrm{mg}$ drospirenone (DRSP) on the first day of menstruation. The pill regimen was followed for six cycles. The 6-month regimen did result in some significant improvements in PCOS symptomatology and hormone levels. Hirsutism was significantly decreased as demonstrated by a significant decrease in F-G score from baseline. Additionally, testosterone levels decreased significantly and SHBG level increased significantly. These two changes also resulted in a significant decrease in the Free Androgen Index of the enrolled women. Overall, this study demonstrated that COCs with DRSP are effective in decreasing hyperandrogenemia and reducing hirsutism (a primary symptom of PCOS).

Guido et al also investigated the effects of a drospirenonecontaining COC in the treatment of PCOS with 18 subjects and 12 cycles of the COC (Guido et al 2004). Similar to Pehlianov and Mitkov, Guido and colleagues observed a significant decrease in hirsutism as demonstrated by a significant decrease in F-G score. This decrease was not seen by the third cycle but was evident at both the 6th cycle and the 12 th cycle. These authors confirmed the previous finding that drospirenone-containing COC does decrease hirsutism in women with PCOS. Guido also noted a significant increase in SHBG at the 3rd cycle, 6th cycle, and 12th cycle. This indicates that the increase in SHBG occurs soon after the administration of the drospirenone-containing COC and that it is a long-lasting rather than transient effect. Guido's results also supported the significant decrease in testosterone found by both Pehlianov and Mitkov, and De Leo and colleagues. These authors noted that the size of the SHBG increase found with drospirenone-containing COC treatment was greater than that found with treatment using $17 \alpha$-hydroxyprogesterone derivatives. This indicates that drospirenone-containing COCs may be more effective in the treatment of hyperandrogenemia 
than COCs that use $17 \alpha$-hydroxyprogesterone derivatives as the progestin agents (Guido et al 2004). A similar study conducted by Batukan and Muderris corroborated these results. Drospirenone-containing COCs were found to significantly increase SHBG levels, significantly decrease testosterone levels, and significantly improve hirsutism after 12 cycles (Batukan and Muderris 2006).

Overall, drospirenone-containing COCs appear to be effective in treating and alleviating the symptoms of PCOS. Numerous studies show a decrease in hirsutism within as few as six cycles of drospirenone-containing COCs. Studies have also consistently demonstrated a decrease in testosterone levels within six cycles and an increase in SHBG in as few as six cycles on therapy. There is also evidence that treatment with a drospirenone-containing $\mathrm{COC}$ decreases the severity of acne in women with PCOS (Palep-Singh et al 2004). Thus, drospirenone-containing COCs are as effective at treating PCOS and its symptoms as other COCs. This could potentially make them more effective in the treatment of PCOS than the traditional COCs.

\section{Metabolic concerns}

More recently, controversy has arisen over the use of COC in patients with PCOS (Nader and Diamanti-Kandarakis 2007) specifically regarding the effects of COCs on carbohydrate metabolism and metabolic parameters such as insulin resistance and glucose tolerance (Korytkowski et al 1995; Nader and Diamanti-Kandarakis 2007). The most significant change found is a deterioration of insulin sensitivity with the administration of COCs (Korytkowski et al 1995; Dahlgren et al 1998). Two studies conducted in obese women with PCOS also showed a decrease in glucose tolerance demonstrated by the results of an oral glucose tolerance test (Nader et al 1997; Morin-Papunen et al 2000). Plasma insulin concentrations were constant in both of these studies indicating that the decrease in glucose tolerance was again due to a decrease in insulin sensitivity rather than a change in insulin levels/production. However, other studies performed in nonobese women showed no change in glucose tolerance and insulin sensitivity (Armstrong et al 2001; Cibula et al 2002; Elter et al 2002; Morin-Papunen et al 2003). This variation in results may suggest that metabolic consequences from COC treatment of PCOS depend on body type (Vrbikova and Cibula 2005).

COC treatment of PCOS also has been shown to cause an increase in total cholesterol, triglycerides, HDL and LDL cholesterol (Creatsas et al 2000; Mastorakos et al 2002; Cibula et al 2005). The opposite finding, however, has been reported with COCs containing the same progestin resulting in an overall decrease in the LDL:HDL ratio (Falsetti and Pasinetti 1995). Triglycerides and HDL cholesterol did increase in both studies however. While cholesterol levels appear to increase in women with PCOS who are treated with COCs regardless of the COC used, desgetrel containing COCs do not seem to cause changes in triglyceride levels while other COCs do (Escobar-Morreale et al 2000; Mastorakos et al 2002).

The metabolic effects of drospirenone-containing COCs are just beginning to be explored. The European Active Surveillance Study on oral contraceptives followed 58,674 women for a total of 142,475 women years of observation and concluded that the risks of adverse cardiovascular disease and other serious events in users of drospirenone-containing oral contraceptives are similar to those associated with the use of other COCs (Dinger et al 2007). Because drospirenone is a less androgenic progestin, the metabolic effects appear to be much less severe or entirely non-existent when women with PCOS are treated with drospirenone-containing COCs. Guido found no significant change in insulin sensitivity in a study of 15 PCOS women treated with drospirenone-containing COCs (Guido et al 2004). The same study also found that drospirenone-containing COCs appear to have the same effect on lipid levels of PCOS women that they do on healthy controls, which is a major improvement from other COCs. A significant increase in triglycerides and HDL cholesterol has been observed but with no shift in the HDL:LDL ratio (Guido et al 2004). Thus, although the metabolic concerns typical of COC administration in healthy women still exist when drospirenone-containing COCs are used in the treatment of PCOS, the use of drospirenone appears to alleviate the metabolic concerns that are specific to women with PCOS.

\section{References}

Armstrong VL, Wiggam MI, et al. 2001. Insulin action and insulin secretion in polycystic ovary syndrome treated with ethinyl oestradiol/cyproterone acetate. QJM, 94:31-7.

Azziz R, Nestler JE, et al. eds. 2006. Androgen excess disorders in women, polycystic ovary syndrome and other disorders. 2nd ed. Totowa, New Jersey: Humana Press

Azziz R, Woods KS, et al. 2004. The prevalence and features of the polycystic ovary syndrome in an unselected population. J Clin Endocrinol Metab, 89:2745-9.

Barth JH, Cherry CA, et al. 1991. Cyproterone acetate for severe hirsutism: results of a double-blind dose-ranging study. Clinic Endocrinol $(O x f)$, 35:5-10.

Batukan C, Muderris II. 2006. Efficacy of a new oral contraceptive containing drospirenone and ethinyl estradiol in the long-term treatment of hirsutism. Fertil Steril, 85:436-40.

Carlstrom K, Karlsson R, et al. 2002. Diurnal rhythm of and effects on oral contraceptives on serum dehydroepiandrosterone sulphate (DHEAS) are related to alterations in serum albumin rather than to changes in adrenocortical steroid secretion. Scand J Clini Lab Invest, 62:361-8. 
Cassidenti DL, Paulson RJ, et al. 1991. Effects of sex steroids on skin 5 alpha-reductase activity in vitro. Obstetrics and Gynecology, 78:103-7.

Cibula D, Fanta M, et al. 2005. The effect of combination therapy with metformin and combined oral contraceptives (COC) versus COC alone on insulin sensitivity, hyperandrogenaemia, SHBG and lipids in PCOS patients. Hum Reprod, 20:180-4.

Cibula D, Sindelka G, et al. 2002. Insulin sensitivity in non-obese women with polycystic ovary syndrome during treatment with oral contraceptives containing low-androgenic progestin. Hum Reprod, 17:76-82.

Creatsas G, Koliopoulos C, et al. 2000. Combined oral contraceptive treatment of adolescent girls with polycystic ovary syndrome. Lipid profile. Ann N Y Acad Sci, 900:245-52.

Dahlgren E, Landin K, et al. 1998. Effects of two antiandrogen treatments on hirsutism and insulin sensitivity in women with polycystic ovary syndrome. Hum Reprod, 13: 2706-11.

De Leo V, Morgante G, Piomboni P, et al. 2007. Evaluation of effects of an oral contraceptive containing ethinylestradiol combined with drospirenone on adrenal steroidogenesis in hyperandrogenic women with polycystic ovary syndrome. Fertil Steril, 88:113-7.

Dinger J, Heinemann L, et al. 2007. The safety of a drospirenone containing oral contraceptive: final results from the European Active Surveillance study on oral contraceptives based on 142,475 women - years of observation. Contraception, 75:344-54.

Elter K, Imir G, et al. 2002. Clinical, endocrine and metabolic effects of metformin added to ethinyl estradiol-cyproterone acetate in non-obese women with polycystic ovarian syndrome: a randomized controlled study. Hum Reprod, 17:1729-37.

Escobar-Morreale HF, Lasuncion MA, et al. 2000. Treatment of hirsutism with ethinyl estradiol-desogestrel contraceptive pills has beneficial effects on the lipid profile and improves insulin sensitivity. Fertil Steril, 74:816-9.

Falsetti L, Pasinetti E. 1995. Effects of long-term administration of an oral contraceptive containing ethinylestradiol and cyproterone acetate on lipid metabolism in women with polycystic ovary syndrome. Acta Obstet Gynecol Scand, 74:56-60.

Guido M, Romualdi D, et al. 2004. Drospirenone for the treatment of hirsute women with polycystic ovary syndrome: a clinical, endocrinological, metabolic pilot study. J Clin Endocrinol Metab, 89:2817-23.

Hammerstein J, Meckies J, et al. 1975. Use of cyproterone acetate (CPA) in the treatment of acne, hirsutism and virilism. $J$ Steroid Biochem, 6:827-36.

Kluft C, Endrikat J, et al. 2006. A prospective study on the effects on hemostasis of two oral contraceptives containing drospirenone in combination with either 30 or $20 \mathrm{mcg}$ ethinyl estradiol and a reference containing desogestrel and $30 \mathrm{mcg}$ ethinyl estradiol. Contraception, 73:336-43.

Knochenhauer ES, Key TJ, et al. 1998. Prevalence of the polycystic ovary syndrome in unselected black and white women of the southeastern United States: a prospective study. J Clinic Endocrinol Metab, 83:3078-82.

Korytkowski MT, Mokan M, et al. 1995. Metabolic effects of oral contraceptives in women with polycystic ovary syndrome. J Clin Endocrinol Metab, 80:3327-34.

Laven JSE, Imani B, et al. 2002. New approach to polycystic ovary syndrome and other forms of anovulatory infertility. Obstet Gynecol Surv, $57: 755-67$.
Legro RS, Driscoll D, et al. 1998. Evidence for a genetic basis for hyperandrogenemia in polycystic ovary syndrome. Proc Natl Acad Sci USA, 95:14956-60.

Lobo RA. 2006. Choice of treatment for women with polycystic ovary syndrome. Fertil Steril, 86(Suppl 1):S22-3.

Mastorakos G, Koliopoulos C, et al. 2002. Androgen and lipid profiles in adolescents with polycystic ovary syndrome who were treated with two forms of combined oral contraceptives. Fertil Steril, 77:919-27.

Mauvais-Jarvis P, Jayle MF, et al. 1965. [In vivo studies on metabolism of androgens following administration of steroid inhibitors of ovulation. 1. Changes in the secretion of androgens and excretion of their metabolites in normal subjects and women with the Stein-Leventhal syndrome]. Acta Endocrinologica, 50:131-44.

Michelmore KF, Balen AH, et al. 1999. Polycystic ovaries and associated clinical and biochemical features in young women. Clin Endocrinol, 51:779-86.

Moghetti P, Tosi F, et al. 2000. Comparison of spironolactone, flutamide, and finasteride efficacy in the treatment of hirsutism: a randomized, double blind, placebo-controlled trial. J Clin Endocrinol Metab, 85:89-94.

Morin-Papunen L, Vauhkonen I, et al. 2003. Metformin versus ethinyl estradiol-cyproterone acetate in the treatment of nonobese women with polycystic ovary syndrome: a randomized study. J Clin Endocrinol Metab, 88:148-56.

Morin-Papunen LC, Vauhkonen I, et al. 2000. Endocrine and metabolic effects of metformin versus ethinyl estradiol-cyproterone acetate in obese women with polycystic ovary syndrome: a randomized study. $J$ Clin Endocrinol Metab, 85:3161-8.

Nader S, Diamanti-Kandarakis E. 2007. Polycystic ovary syndrome, oral contraceptives and metabolic issues: new perspectives and a unifying hypothesis. Hum Reprod, 22:317-22.

Nader S, Riad-Gabriel MG, et al. 1997. The effect of a desogestrel-containing oral contraceptive on glucose tolerance and leptin concentrations in hyperandrogenic women. J Clin Endocrinol Metab, 82:3074-7.

Palep-Singh M, Mook K, et al. 2004. An observational study of Yasmin in the management of women with polycystic ovary syndrome. J Fam Plann Reprod Health Care, 30:163-5.

Pehlivanov B, Mitkov M. 2007. Efficacy of an oral contraceptive containing drospirenone in the treatment of women with polycystic ovary syndrome. Eur J Contracept Reprod Health Care, 12:30-5.

Pugeat M, Nicolas MH. et al. 1993. Androgens in polycystic ovarian syndrome. Ann N Y Acad Sci, 687:124-35.

van der Vange N, Blankenstein MA. et al. 1990. Effects of seven low-dose combined oral contraceptives on sex hormone binding globulin, corticosteroid binding globulin, total and free testosterone. Contraception, $41: 345-52$

Venturoli S, Marescalchi O, et al. 1999. A prospective randomized trial comparing low dose flutamide, finasteride, ketoconazole, and cyproterone acetate-estrogen regimens in the treatment of hirsutism. $J$ Clin Endocrinol Metab, 84:1304-10.

Vrbikova J, Cibula D. 2005. Combined oral contraceptives in the treatment of polycystic ovary syndrome. Hum Reprod Update, 11:277-91.

Wiegratz I, Kutschera E, et al. 2003. Effect of four different oral contraceptives on various sex hormones and serum-binding globulins. Contraception, 67:25-32. 